\title{
Mesenchymal Stem Cells: A New Approach for Treating Bronchopulmonary Dysplasia
}

\author{
Shamimunisa B Mustafa* \\ Department of Pediatrics/Division of Neonatology University of Texas Health San Antonio, USA
}

*Corresponding author: Shamimunisa B Mustafa, Department of Pediatrics/Division of Neonatology University of Texas Health San Antonio, 7703 Floyd Curl Drive, MSC 7812 San Antonio, TX 78229-3900, USA.

To Cite This Article: Shamimunisa B Mustafa. Mesenchymal Stem Cells: A New Approach for Treating Bronchopulmonary Dysplasia. Am J Biomed Sci \& Res. 2019 - 5(5). AJBSR.MS.ID.000947. DOI: 10.34297/AJBSR.2019.05.000950.

Received: 眥October 04, 2019; Published: 盋October 14, 2019

\begin{abstract}
In spite of improved clinical management premature babies are highly susceptible to several neonatal related-morbidities, namely respiratory, neurological, and gastrointestinal. Bronchopulmonary dysplasia (BPD) is the most common lung disorder in premature babies. Present therapeutic options are mainly supportive and do not completely cure BPD, mainly because of its multifactorial pathogenesis. Mesenchymal stem/stromal cells (MSCs) have materialized as a highly promising therapeutic option for alleviating BPD. The many facets of this novel therapy are discussed in this review.
\end{abstract}

Keywords: Prematurity; Bronchopulmonary dysplasia; Mesenchymal stem/stromal cells; Lung disease

Abbreviations: BPD: Bronchopulmonary Dysplasia; MSCs: Mesenchymal stem/stromal cells; IUGR: Intrauterine Growth Restriction; CPAP: Continuous Positive Airway Pressure; PPV: Positive Pressure Ventilation; PDA: Patent ductus Arteriosus.

\section{Introduction}

Worldwide approximately 15 million babies are born prematurely before 37 weeks gestational age, of which an estimated 1 million of these babies die due to complications directly associated with preterm delivery [1]. Premature babies are highly susceptible to several neonatal related-morbidities, namely respiratory, neurological, and gastrointestinal [2]. Several of these illnesses can persist past infancy and childhood and ultimately become a considerable financial burden to both families and healthcare systems [3]. A type of chronic lung disease, bronchopulmonary dysplasia (BPD), particularly prevalent in preterm babies that had received supplemental oxygen and supported by mechanical ventilation for alleviating respiratory failure was first communicated in 1967 [4]. At that time, BPD manifested itself as lung injury with marked inflammation and fibrosis [4]. Since then, significant progress in perinatal and neonatal medicine, together with innovative practices, and procedures have greatly improved survival of extremely premature babies born as early as 22-24 weeks gestational age [5]. At the same time, the incidence of BPD has increased; $45 \%$ of preterm babies that are delivered between
22-27 weeks gestational age will go on to develop BPD; in the United States there are up to 10,000 reported incidents of preterm babies with BPD each year [5].

Concurrent with these changes, the pathophysiology of BPD has altered and is now characterized by reduced alveolarization, impaired development of blood vessels and the microvasculature, enlarged airspaces, and poor lung function [6]. The etiology of BPD in the preterm baby is multifactorial; the extent of prematurity, several antenatal insults, and anomalies followed by postnatal influences and other co-morbidities which occur as a consequence of preterm birth all contribute to the onset and progression of BPD, summarized in Figure 1 [6-8]. Anomalies in lung function that began at birth owing to prematurity, subsequent extrauterine adaptations, and the manifestation of BPD persist throughout life [9, 10]. Studies have reported that children formerly born premature with BPD were more likely to exhibit poor lung function by the time they reached school-age [10]. Long-term follow-up studies have also reported sub-optimal lung function and increased incidence of emphysema in young adults who were former BPD patients $[11,12]$. 


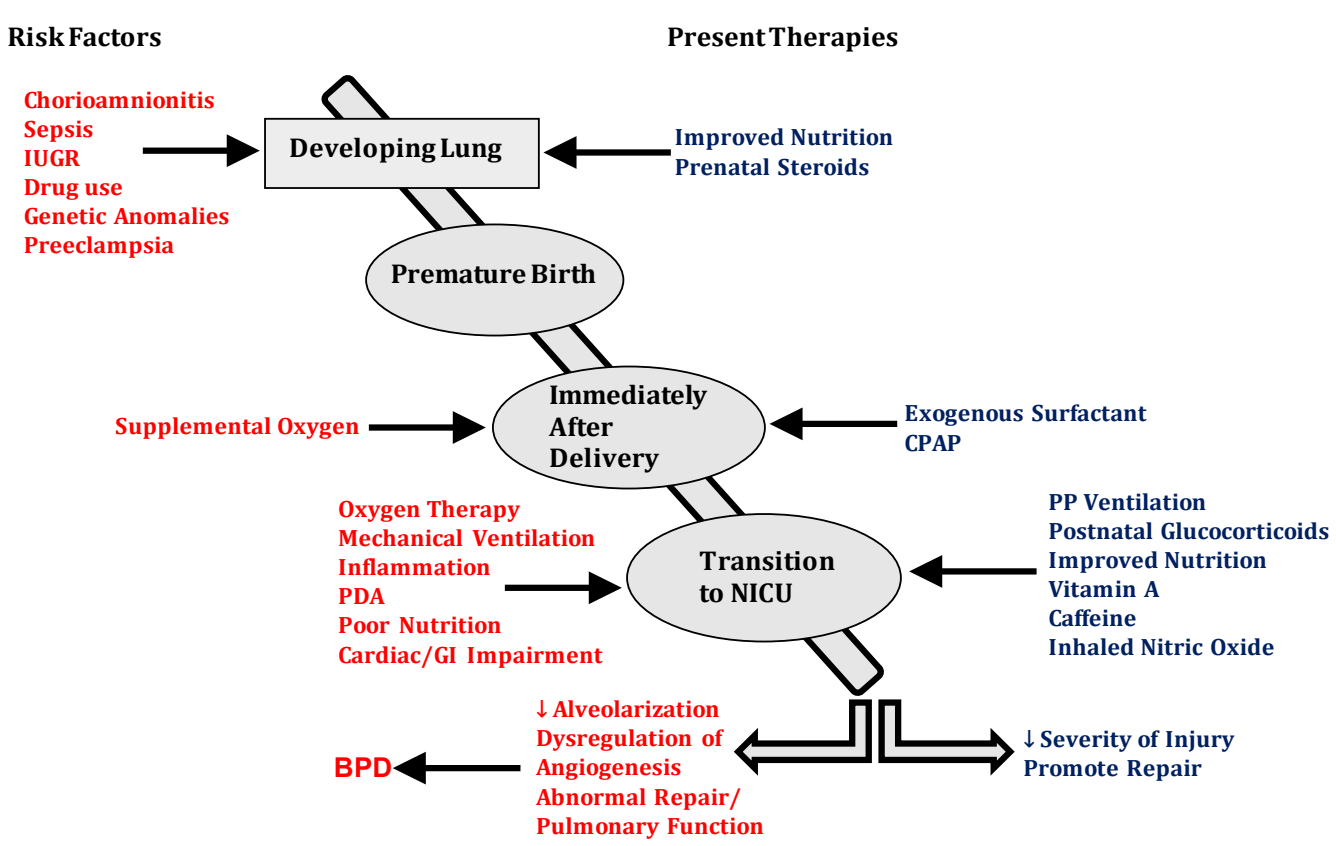

Figure 1: Left, The multifactorial risk factors of bronchopulmonary dysplasia. Right, Present therapeutic options and clinical strategies with the aim to reduce the severity of bronchopulmonary dysplasia.

\section{Existing Therapeutic Options for BPD}

Despite considerable advances in neonatal clinical management BPD persistently presents itself as a significant illness for premature babies. As the etiology of BPD is multifactorial this presents a confounding therapeutic conundrum. In the ideal setting prevention of premature birth is the sole solution to avoiding the onset of BPD; a feat that has yet to be achieved. Current therapies are mainly supportive and directed towards minimizing lung injury (summarized in figure 1), but neither significantly diminish the incidence of BPD nor do they alter the pathophysiological course of the disease process [13-18]. In follow-up studies, certain treatment regimens such as postnatal dexamethasone has been associated with adverse neurological outcomes [19]. Clearly, there is a pressing requirement to identify alternative therapies that are both effective and safe for alleviation of this debilitating, multifactorial lung disease.

\section{An Untapped Therapeutic Option: Mesenchymal Stromal/Stem Cells}

Bone marrow-derived mesenchymal stromal/stem cells (MSCs) were first described by Friedenstein and colleagues and their intrinsically diverse properties, attributes (e.g. self-renewal, differentiation, pro- angiogenic, anti-inflammatory, anti-fibrotic, and antioxidant), and classifications have been previously reviewed [20-22]. MSCs have been identified in almost all fetal and adult tissues and play crucial roles in promoting tissue development and the reparative responses to the injured host tissues/cells [21,23,24]. In the fetal lung resident or endogenous MSCs coordinate and foster alveolar development, tissue reparative processes, and growth of the pulmonary vasculature [23,25]. Recently Collins and colleagues reported that the repair potential of resident fetal lung MSCs isolated from an oxygen-induced rat BPD model was altered [26]. In addition, studies by Popova and colleagues showed that cultured MSCs isolated from tracheal aspirates of preterm babies that subsequently developed BPD exhibited a myofibroblast phenotype suggesting these cells exhibit a dual mode of action that is dependent upon their environment; Specifically, under normal circumstances resident lung MSCs promote lung growth, repair and development, whereas under constant injury these cells very likely switch to a pathogenic pathway [27].

Owing to the diverse properties and attributes of MSCs coupled with experimental evidence describing their dysfunction in BPD, sound judgement prevails that exogenous MSCs will be a suitable alternative treatment option for BPD. To further support the utility of MSCs as a therapeutic contender for BPD, several logistical aspects have to be considered:

a) MSCs can be easily isolated from adipose and bone marrow tissue in sufficient amounts [28]. MSCs can also be isolated devoid of ethical constraints from placental and umbilical cord tissue (Wharton's jelly) and blood, and amniotic fluid which provides an autologous source of cells [29,30]. Regardless of the source, MSCs can be easily cultured and rapidly expand in carefully controlled environments, essentially following good manufacturing practices;

b) MSCs do not express human leukocyte antigen class II which affords allogenic treatment [31]; and

c) MSCs can move towards injured tissues and selectively adapt their reparative actions [32].

At first, it was postulated that MSCs exerted their beneficial effects by migrating to the site of injury, followed by engraftment, and then differentiating in to the compromised/damaged cells [34]. Subsequent studies in rodent models of BPD reported poor rates of engraftment of exogenous MSCs coupled with the finding that 
MSCs only lasted for a few days in the lung [34-36]. The accepted means by which MSCs exert their beneficial effects is via paracrine mechanisms. This includes secretion of bioactive substances with anti- apoptotic, anti-inflammatory, and pro-angiogenic properties (collectively referred to as the secretome or conditioned media) within the microenvironment and exosomes, containing proteins, microRNAs, and mRNA fragments [37,38]. Exosomes are then taken up by the damaged cells by means of vesicle fusion [40]. Also, MSCs can transfer mitochondria to target cells through nanotubes or microvesicles [37]. This is particularly beneficial to minimize apoptosis in target cells [37].

\section{Therapeutic Effects of MSCs: Proving a Concept}

The conviction that exogenous MSCs and or the secretome will be beneficial in ameliorating BPD-like lung damage in experimental animal models was validated by Augustine and colleagues, who completed a systematic review and meta-analysis study [41]. In this study the authors categorized 25 independent research reports which utilized newborn rodents (mice and rats) who were exposed to hyperoxia and displayed BPD-like lung injury. Animals received either human umbilical cord or cord blood or rat bone marrow derived MSCs which were administered either via intraperitoneal, intravenous, or intratracheal routes [41]. Variables in these studies included source and route of administration of MSCs, the number of administered cells, and long-term outcomes. In the MSCs-treated rodents, significant improvements in lung function and alveolarization, and vascular growth coupled with decreased inflammation and oxidative stress was observed [7,41-43].

\section{Clinical Trials: Taking the Concept to the Bedside}

The outcomes of the initial, groundbreaking phase I clinical trial was recently reported [44]. In this study, nine preterm babies (23-29 weeks gestational age) requiring mechanical ventilation between 5-14 days following birth were treated once with either $10^{7}$ or $2 \times 10^{7}$ umbilical cord blood derived MSCs (intratracheal delivery). It is extremely important to note that these babies did not exhibit any undesirable consequences thus strongly supporting the safety and practicality of this novel therapeutic modality [44]. The same investigators went on to report that there were no indications of neurological, respiratory, or growth deficits in these babies after 2 years [45]. These pioneering studies have set the precedence for several other independent investigators to initiate their own phase I-II trials, summarized in Table 1.

\begin{tabular}{|c|c|c|c|c|c|}
\hline NCT ID number & Phase & Cell Source & Administration & Number of Enrollees & $\begin{array}{l}\text { Age of Enrollees } \\
\text { (Upper Limit) }\end{array}$ \\
\hline NCT01207869 & I & UC-MSC & Intra-tracheal & 10 & $6 \mathrm{mos}$ \\
\hline NCT01297205 & I & Allogenic UCB-MSC & Intra-tracheal & 9 & 14 days \\
\hline NCT01632475 & I & Allogenic UCB-MSC & Intra-tracheal & 9 & $48 \mathrm{mos}$ \\
\hline NCT02023788 & I & Allogenic UCB-MSC & Intra-tracheal & 8 & $63 \mathrm{mos}$ \\
\hline NCT02443961 & I & MCS & Not defined & 10 & 28 wks \\
\hline NCT03378063 & I & Allogenic UCB-MSC & Not defined & 100 & $3 \mathrm{mos}$ \\
\hline NCT03631420 & I & UC-MSC & Not defined & 9 & 38 wks \\
\hline NCT03683953 & I & MSC & Intra-tracheal & 200 & 37 wks \\
\hline NCT03857841 & $\mathrm{I}$ & BM-MSC EV & Intravenous & 18 & 14 days \\
\hline NCT03873506 & I & UC-MSC & Intravenous & 30 & $5 \mathrm{yrs}$ \\
\hline NCT02381366 & I-II & Allogenic UCB-MSC & Not defined & 12 & 14 days \\
\hline NCT03558334 & $\mathrm{I}-\mathrm{II}$ & UCB-MSC & Intravenous & 30 & Not defined \\
\hline NCT03645525 & I-II & UC-MSC & Intra-tracheal & 180 & 3 wks \\
\hline NCT03774537 & I-II & UC-MSC & Intravenous & 20 & 14 days \\
\hline NCT01828957 & II & Allogenic UCB-MSC & Intra-tracheal & 70 & 14 days \\
\hline NCT01897987 & II & Allogenic UCB-MSC & Intra-tracheal & 70 & $7 \mathrm{mos}$ \\
\hline NCT03392467 & II & Allogenic UCB-MSC & Not defined & 60 & 13 days \\
\hline NCT03601416 & II & UC-MSC & Intravenous & 57 & $12 \mathrm{mos}$ \\
\hline
\end{tabular}

\section{Conclusion}

Identifying the most effective treatment for BPD has indeed proven an onerous task. However, owing to advancements in MSCbiology has now afforded us with a potentially very promising therapeutic option. Preclinical studies using MSCs for the treatment of BPD have essentially paved the way for the initiation of several clinical trials. Of note, given the multifactorial nature of BPD, one has to consider that MSCs combined with other drugs may yet prove to be an additional useful therapeutic option. Concurrent with the clinical trials, we must still continue to investigate the long-term efficacy and safety of this particular therapy. Also, of importance is to 1) further understand the mechanistic pathways of MSCs mode 
of action using both in vivo and in vitro models; and 2) investigate the effects of the microenvironment on the biological properties of lung tissue resident/endogenous MSCs. It is unknown as to whether endogenous fetal lung-MSCs recover and regain their biological properties after cessation or lessening of BPD or how for long they remain in a quiescent/altered state. In a similar context, although recent studies have clearly demonstrated the therapeutic efficacy of exogenously applied MSC or their secretome via a paracrinemediated effect in animal models of neonatal lung disease, the interaction between exogenous MSCs and compromised resident fetal lung-MSCs is unclear.

\section{References}

1. March of Dimes (2012) PM NCH, Save the Children WHO. Born Too Soon: The Global Action Report on Preterm Birth. Geneva: WHO, Switzerland.

2. Manuck TA, Rice MM, Bailit JL, et al. (2016) Preterm neonatal morbidity and mortality by gestational age: A contemporary cohort. Am J Obstet Gyn 215: 103-114.

3. Saigal S, Doyle, LW (2008) An overview of mortality and sequelae of preterm birth from infancy to adulthood. Lancet 371: 261-269.

4. Northway WH, Rosan RC, Porter DY (1967) Pulmonary disease following respiratory therapy of hyaline-membrane disease: Bronchopulmonary Dysplasia. N Engl J of Med 276: 357-368.

5. Stoll BJ, Hansen NI, Bell EF (2015) Trends in care practices, morbidity \& mortality of extremely preterm neonates. JAMA 314(10): 1039-1059.

6. Alvira CM, Morty RE (2017) Can we understand the pathobiology of Bronchopulmonary Dysplasia. J Ped 190: 27-37.

7. Alvarez-Fuente M, Moreno L, Mitchel JA, Reis IK, Lopez P, et al. (2018) Preventing bronchopulmonary dysplasia: New Tools for an old challenge. Ped Res 85(4): 432-441.

8. Higgins RD, Jobe AH, Thomas-Kosa M, Bancala E, Viscard Rm, et al. (2018) Bronchopulmonary Dysplasia: Executive Summary of a Workshop. J Pediatr 207: 264-273.

9. Stocks J, Hislop A, Sonnappas (2013) Early Lung development: Lifelong effect on respiratory health and disease. Lancet Respir Med 1(9): 728742 .

10. Islam, JY, Keller RL, Aschner JL, Hartert TV, Moore PE (2015) Understanding the Short- and Long- term respiratory outcomes of prematurity and bronchopulmonary dysplasia. Am J Resp Crit Care Med 192(2): 134-156

11. Von Hove MP, Uhlig HH, Robert-Tllig (2014) Pulmonary Outcomes in former Preterm, Very Low Birth Weight Children with BPD: A case control follow-up at school age. J Pediatr 164(1): 40-45.

12. Gough A, Linden M, Spence D, Patterson CC, Halliday H et al. (2014) Impaired lung function \& health status in adult survivors of BPD. Eur Resp J 43: 808-816.

13. Tarnow Mordi W (2016) Outcomes of two trials of oxygen - saturation targets in preterm infants. N Grg J Med 374: 749-760.

14. Bahadue Fl, Soll R (2012) Early versus delayed selective surfactant treatment for neonatal respiratory distress syndrome. Cochrane Database Syst Rev 11: CD001456.

15. Schmidt B (2006) Caffeine therapy for apnea of prematurity. New Eng J Med 354: 2112-2121.

16. Darlaw BA, Graham PJ (2011) Vit A supplementation to prevent mortality short- and Long-term morbidity in very low birthweight infants. Cochrane database syst Rev 10: Cd000501.

17. Donohue PK (2014) Inhaled nitric oxide in preterm infants: a systematic review. Pediatrics 127(2): 414-427.
18. Doyle LW, Ehrenkranz RA, Halliday HL (2014) Early (< 8 days) postnatal corticosteroids for preventing chronic lung disease in preterm infants. Cochrane database Systematic Review 5: CD001146.

19. Wilson-Costello D (2009) Impact \& postnatal corticosteroid use on neurodevelopment at 18-22 months' adjusted age: effects of dose, timing, and risk of BPD in extremely low birthweight infants. Pediatrics 123(3): 430-437.

20. Friedenstein AJ, ChailKhjan Rk, Laly Kina KS (1970) The development of fibroblast colonies in monolayer cultures of guinea-pig marrow \& spleen cells. Cell Tissue Kinet 3(4): 393-403.

21. Mobius MA, Thebaud B (2015) Stem Cells and their mediators-next generation therapy for bronchopulmonary dysplasia. Frontiers in Medicine 2: 50-62.

22. Caplan Al, Correa D (2011) The MSC: an injury drug store. Cell Stem Cell 9(1): 11-15.

23. Collins JJ and Thebaud B (2014) Lung mesenchymal stromal cells in development and disease: To serve and protect. Antioxid Redox Signal 21(13): 1849-1862.

24. Meirelles Lda, Fontes Am, Covas DT, Capllan Al (2009) Mechanisms involved in the therapeutic properties of mesenchymal stem cells. Cytokine Growth Factor Rev 20(6): 419-427.

25. Mobius MA \& M Rudiger (2016) Mesenchymal stromal cells in the development and therapy of BPD. Mol Cell Pediatrics 3(1): 18-26.

26. Collins, JJP, Lithopoulos MA, Dos Santos CC, Issa N (2018) Impaired angiogenic supportive capacity and altered gene expression profile of resident $\mathrm{CD} 146^{+}$mesenchymal stromal cells isolated from hyperoxiainjured neonatal rat lungs. Stem Cells and Dev 27(16): 1109-1124.

27. Popova AP, Bozyk PD, Bentley JK (2010) Isolation of tracheal aspirate mesenchymal stromal cells predicts bronchopulmonary dysplasia. Pediatrics 126(5): 1127-1133.

28. Romanov YA, Darevskaya AN, MerzliKina NV, Buravkova LB (2005) Mesenchymal stem cells from human bone marrow and adipose tissue: isolation, characterization and differentiation potentialities. Exp Bio Med 140(1): 138-143

29. In't Anker PS, Scherjan SA, Kleijburg KC, DeGroot-Swings GM, Class FH, et al. (2004) Isolation of mesenchymal stem cells of fetal or maternal origin from human placenta. Stem Cells 22(7): 1338-1345.

30. Wang HS, Hung SC, Peng ST, Huang CC, Wei HM, et al. (2004) Mesenchymal stem cells in the Wharton's jelly of the human umbilical cord. Stem Cells 22(7): 1330-1337.

31. Dominici M, Le Blanc K, Mueller I, Slaper-Cortenbach I, Marini C, et al. (2006) Minimal criteria for defining multipotent mesenchymal stromal cells. The International Society for Cellular Therapy Position Statement, Cytotherapy 8: 315-317.

32. Sackstein R, Merzban JS, Cain DW, Dagia NM, Spencer JA, et al. (2008) Ex Vivo glycan engineering of CD44 programs human multipotent mesenchymal stem cells trafficking to bone. Nat Med 14(2): 181-187.

33. Laube M, Stolzing A, Thorne UH, Fabian C (2016) Therapeutic potential of mesenchymal stem cells for pulmonary complications associated with preterm birth. Int J Biochem Cell Biol 74: 18-32.

34. Van Haaften T, Byrne R, Bonnet S (2009) Airway delivery of mesenchymal stem cells prevents arrested alveolar growth in neonatal lung injury in rats. Am J of Respir and Crit Care Med 180(11):1131-1142.

35. Aslam M, Baveja R, Liang O (2009) Bone marrow stromal cells attenuate lung injury in a murine model of neonatal chronic lung disease. Am J of Respir and Crit Care Med 180: 1122-1130.

36. Kotton DN, Fabian AJ, Mulligan RC (2005) Failure of bone marrow to reconstitute lung epithelium. Am J Respir Cell Mol Biol 33: 328-334.

37. Spees JL, Love RH \& Gregory CA (2016) Mechanisms of mesenchymal stromal/stem cell function. Stem Cell Res. \& Ther. 7: 125-138. 
38. Fung ME, Thebaud B (2014) Stem cell-based therapy for neonatal lung disease: it's in the juice. Peds Res 75: 2-7.

39. Sdrumas K, Kourembanas S (2014) MSC microvesicles for the treatment of lung disease: a paradigm for cell free therapy. Antioxid Redox Sig 21(13): 1905-1915.

40. Stoorvogel W (2012) Functional transfer of micro RNA by exosomes. Blood 119: 646-648.

41. Augustine S, Avey MT, Harrison B, Lock T, Ghannad M, et al. (2017) Mesenchymal stromal cell therapy in bronchopulmonary dysplasia. Systematic Review \& Meta-Analysis of Preclinical Studies. Stem Cells Trans Med 6: 2079-2093.
42. ThebaudB (2019) Stem cells for extreme prematurity. Am J Perinatal 36: 568-573.

43. Nitkin CR, Rajasingh J, Pisano C, Besner GE, Thebaud B, et al. (2019) Stem cell therapy for preventing neonatal disease in the 21st century: Current Understanding and Challenges. Peds Res 6: 1-12.

44. Ahn SY, Chang YS, Kim JH, Sung SI, Park WS (2017) Two-Year follow up outcomes of premature infants enrolled in the phase 1 trial of mesenchymal stem cells transplantation of bronchopulmonary dysplasia. J Peds 185: 49-54.

45. Chang YS, Ayn SY, Yoo HS (2014) Mesenchymal stem cells for bronchopulmonary dysplasia: Phase 1 dose - escalation clinical trial. J Peds 164: 966-972. 